\title{
Economie rurale non agricole, levier de réduction de la pauvreté dans le Bassin arachidier du Sénégal
}

\author{
Amadou TANDJIGORA et Thierno Bachir SY \\ Université Cheikh Anta Diop, Dakar, Sénégal
}

\begin{abstract}
Résumé : Cet article analyse par des faits stylisés les activités non agricoles ${ }^{1}$ qui sont classifiées sous forme d'industries et de services ruraux. Une étude comparative sur le niveau de pauvreté entre les ménages agricoles et les ménages non agricoles a été faite. Les résultats ont ainsi montré que parmi les individus de l'échantillon qui se sont déclarés pauvres, $72 \%$ exerçaient l'agriculture et les métiers connexes comme principale activité et $28 \%$ exerçaient les activités non agricoles. Le ratio « pauvre/non pauvre » du secteur agricole évalué à 3,17 traduit toute la difficulté de l'agriculture bien qu'elle continue d'employer le maximum de ménages en zone rurale. Ce ratio est au même moment égal à 1,30 pour les industries rurales et 1,27 pour les services, ce qui signifie en outre que les pratiquants des industries et services ruraux sont moins sensibles à une insuffisance de ressources monétaires, comparé aux agriculteurs.
\end{abstract}

Mots clés : Pauvreté rurale, agriculture, industries, services, activités non agricoles, économie rurale non agricole, Bassin arachidier, Sénégal.

\section{Introduction}

L'extension de la pauvreté est le phénomène qui a sans doute le plus marqué la société et l'économie sénégalaise au cours des dernières décennies. Ainsi, la forte pression démographique, la sécheresse, les interventions directes et indirectes dans le secteur agricole, la faiblesse des disponibilités alimentaires combinée à la stagnation ou pire à la chute de la production et des revenus agricoles, mais également aux politiques d'ajustement structurel, ont été quelques causes majeures de la dégradation des conditions de vie des populations rurales et l'expansion de la pauvreté (Diagana, 2008 ; Cabral, 2003). Cependant, la dévaluation du francs CFA, intervenue en janvier 1994, a certes atténué la chute des revenus réels des populations rurales, mais n'a pas infléchi cette tendance à la baisse. C'est dans ce contexte qu'en 2002, le Sénégal a adopté un Document de Stratégie de Réduction de la Pauvreté-DSRP dont l'un des principaux axes est l'allocation au secteur rural de la plus grande partie des ressources publiques mobilisées. Cette orientation de la politique économique selon (Cabral et al, 2003), vise ainsi à accroitre les revenus des pauvres et attaquer la pauvreté là où elle est principalement localisée.

En effet, des auteurs comme Madaki et Adefila (2014) dans leur étude sur la contribution des activités rurales non agricoles au revenu des ménages du Nigéria, affirment qu'il est universellement accepté que le secteur agricole soit incapable de créer suffisamment d'opportunités d'emplois rémunérateurs au milieu d'une population croissante dans les pays en développement. Ce, malgré qu'elle représente la principale activité économique des ménages ruraux presque partout dans les pays pauvres. Leur position est plus ou moins en phase avec les travaux de Katega (2013). Dans son article sur « les activités non

\footnotetext{
${ }^{1}$ Activité non agricole $=$ Economie rurale non agricole
} 
agricoles en milieu rural et la réduction de la pauvreté en Tanzanie », il s'aperçoit qu'entre 60 et $80 \%$ des revenus des ménages ruraux en Afrique Sub-saharienne proviennent des activités non agricoles.

Au Sénégal, l'agriculture occupe près de $60 \%$ des actifs, mais ne parvient tout de même pas à stabiliser le revenu des ménages, encore moins à les sortir de la précarité. Au même moment, de nombreux autres actifs sont occupés par des activités non agricoles comme activité principale ou comme activité secondaire. En effet, il semble opportun de sortir de l'intuition et de comparer le niveau de pauvreté des deux secteurs. Ce qui amène à se poser comme principale question : quelle est entre l'agriculture et les activités non agricoles, l'activité la plus sensible à la pauvreté.

L'objectif principal de cette étude est de montrer parmi l'agriculture et les industries et services ruraux, l'activité économique qui enregistre le taux de pauvreté le plus élevé. Autrement dit, quelle est l'activité économique qui présente le ratio « pauvre/non pauvre » le plus élevé. Parallèlement, le secteur d'activité qui présente le ratio «pauvre/non pauvre » le plus faible serait plus favorable économiquement aux ménages et plus apte à les écarter de la pauvreté.

La première partie de cet article sera consacrée à la présentation de zone d'étude. La deuxième partie traite la revue théorique de la littérature sur la pauvreté dans le monde rural de façon générale. La méthodologie sera élucidée dans la troisième partie. Les résultats et discussions seront présentés respectivement dans la quatrième et cinquième partie.

\section{Présentation de la zone du Bassin arachidier}

Le Bassin arachidier est la principale zone agroécologique du Sénégal. Elle est composée de neuf régions administratives (Thiès, Kaolack, Kaffrine, Fatick, Diourbel, Louga, Tambacounda, Kolda et Sédhiou). Les trois dernières citées ont rejoint la zone agroécologique en raison d'un glissement progressif de la production de l'arachide. La population des régions du Bassin arachidier représente 57,37\% de la population sénégalaise (ANSD,2013). Les ethnies les plus représentées sont les wolofs et les sérères respectivement, la première et la troisième langue nationale du Sénégal.

\subsection{Carte de situation du Bassin arachidier}

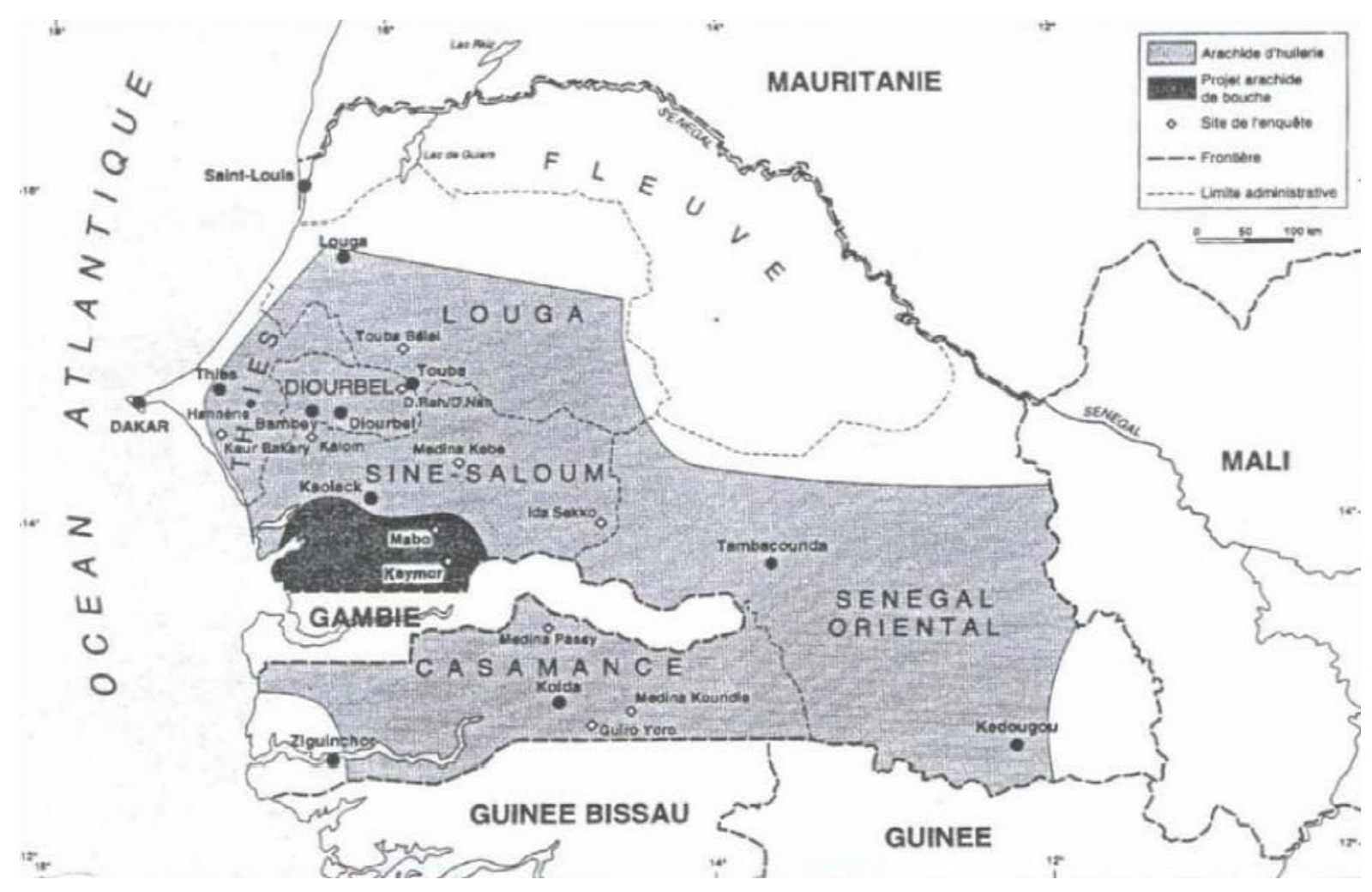

Source : CIRAD (2005) 


\subsection{L'économie de la Zone Agro écologique du Bassin arachidier}

L'économie du Bassin arachidier est particulièrement centrée autour de trois types de sources de revenus à savoir l'agriculture, l'économie non agricole et la migration. D'abord, l'agriculture représente l'activité économique principale des populations de la zone. Selon le rapport sur le Recensement Général de la Population et de l'Habitat de l'Agriculture et de l'Elevage-RGPHAE réalisé en 2013, 65\% des ménages agricoles du pays sont situés dans la zone agroécologique du Bassin arachidier et 83,3\% de ces derniers sont des ruraux. Le mil y représente la principale culture vivrière et l'arachide la principale culture de rente. Cette dernière assure selon l'ISRA (2008), une bonne partie du revenu monétaire des paysans.

Par ailleurs, les petites activités non agricoles font leur apparition dès les premières heures de la culture de l'arachide dans le bassin avec l'usage des charrettes pour transporter des personnes et des bagages au cours des marchés hebdomadaires, moyennant une somme pas assez conséquente à la fin de la journée. Ces activités non agricoles ont commencé leur ascension suite aux diverses contraintes qui pesaient sur l'agriculture, mais les revenus des pratiquants étaient maigres et leur permettaient à peine de survivre. Les ressources tirées de ces activités n'étaient pas très conséquentes et remédiaient faiblement aux problèmes financiers et alimentaires des agriculteurs (Diop, 1992). Aujourd'hui, elles sont plus développées et plus utiles puisque plus de 30\% des actifs de la zone agro écologique les adoptent comme principale activité.

La dernière source de revenus qu'est la migration n'est cependant pas une activité économique puisqu'elle n'est réellement pratiquée dans la zone agroécologique. Elle constitue tout de même une source de revenus dont une partie sera au bénéfice des parents restés sur place, mais aussi du ménage pour la consommation alimentaire.

\section{Revue de la littérature}

Cette étape est primordiale pour établir l'état des lieux des travaux scientifiques sur la pauvreté rurale. Elle a ainsi nécessité d'une part, un tour d'horizon sur la pauvreté rurale en Afrique. D'autre part, elle a établi une analyse de la situation dans le bassin arachidier du Sénégal.

\subsection{Vue d'ensemble sur la pauvreté rurale en Afrique}

La part de la population africaine vivant dans l'extrême pauvreté a considérablement diminué. Elle a chuté de 54\% en 1990 à $41 \%$ en 2015 . Les africains sont en effet plus nombreux à vivre dans la pauvreté aujourd'hui qu'en 1990, à cause en partie de la croissance démographique. Les individus les plus pauvres sont de plus en plus concentrés en Afrique (Beegle et Christiaensen, 2019a). Les mêmes auteurs ont affirmé en 2016 dans leur document portant sur l'évolution de la pauvreté dans une Afrique en plein essor, que les défis de la pauvreté et de la prospérité inclusive demeurent énormes et que dans de nombreux pays africains, les taux de pauvreté sont les plus élevés du monde et devraient rester à deux chiffres.

Ainsi, selon Allen et al (2018), cités par Beegle et Christiaensen (2019b), la plupart des pauvres en Afrique (82\%) vivent dans les zones rurales et tirent principalement leurs moyens de subsistance de l'exploitation agricole, soit $70 \%$ des revenus des pauvres ruraux. Par ailleurs, malgré la croissance économique en Afrique, la croissance démographique rapide et persistante de la région, ses entraves structurelles (faible capital humain, inégalité persistante entre les sexes, et graves déficits d'infrastructure) et sa dépendance croissante vis-à-vis des ressources naturelles continuent de freiner la réduction de la pauvreté. D'après le rapport 2010 du Fonds International pour le Développement de l'Agriculture-FIDA, la croissance de la population rurale ralentit, mais les chiffres ne baisseront pour l'Afrique Sub-saharienne que vers 2045.

Dans ce cas précis, le développement de ces zones rurales devrait être l'axe prioritaire pour la réduction de la pauvreté dans ces pays. Toutefois, la création de nouvelles possibilités de réduction de la pauvreté 
rurale et de la croissance économique exige une approche diversifiée du développement rural englobant bien entendu l'agriculture, mais aussi l'économie rurale non agricole.

Au Sénégal, ces dernières décennies sont marquées principalement par l'extension de la pauvreté, de plus en plus galopante. En effet, la paupérisation des populations rurales est induite par la forte pression démographique, les effets néfastes du changement climatique. Il ressort de ce dernier mentionné, une faible disponibilité de ressources alimentaires combinant à la stagnation voire une chute de la production et des revenus agricoles. De même, sur le plan politique, il est souvent constaté des interventions directes et indirectes dans le secteur agricole à l'instar des politiques d'ajustement structurel qui ont contribué à la dégradation des conditions de vie des ruraux ainsi que l'expansion de la pauvreté, y compris en zone urbaine (Diagana, 2008 ; Cabral, 2003).

Au demeurant, le taux de pauvreté au Sénégal est globalement évalué à $46,7 \%$ au niveau national et $57,3 \%$ en milieu rural (ANSD, 2013). A l'échelle des ménages, $69 \%$ des ruraux se considèrent pauvres dont $53,2 \%$ se déclarent très pauvres (ANSD, 2014). Cette situation met en exergue une ruralisation du phénomène de la pauvreté dans ce pays marqué par l'importance des espaces ruraux agricoles à l'instar du Bassin arachidier.

\subsection{Analyse de la pauvreté dans le Bassin arachidier du Sénégal}

Dans la plupart des pays en développement, une masse importante de ménages ou d'individus pauvres est employée par le secteur agricole. Le rapport 2015 de la Banque africaine de développement sur la croissance, la pauvreté et l'inégalité révèle que $54 \%$ des pauvres en Afrique sont actifs dans l'agriculture. Au Sénégal, particulièrement dans le Bassin arachidier, 69\% des actifs occupés des deux secteurs agricoles et non agricoles s'étaient déclarés pauvres en 2005. Il s'aperçoit ainsi que plus de $72 \%$ des actifs pauvres occupaient l'agriculture et ses métiers connexes comme principale activité et source de revenus. En effet, le changement climatique, combiné à une dégradation des sols due au manque de jachère, met les ménages de cette zone arachidière face à des risques majeurs, surtout les plus pauvres d'entre eux (Faye et al. 2019).

Les risques sont encore plus marqués pour 1'indisponibilité des ressources notamment celles alimentaires. A ce titre, selon l'ANSD (2014) 65,8\% des ménages en milieu rural ont peur de manquer de nourriture dont, $57 \%$ ont vécu la faim du fait de l'absence et de l'insuffisance des ressources. Cet état de fait est foncièrement établi dans le Bassin arachidier sénégalais où $90 \%$ des ménages étaient répertoriés en 2016 sous le niveau moyen national de pauvreté.

Cette conjoncture fait du Bassin arachidier l'une des plus importantes zones de départ pour l'exode rural qui semble devenir structurel et permanent. Cette migration rurale charrie des opportunités pour certains, devenant un fondement du tissu économique qui les retient au terroir (Ndao, 2017). Cet exemple vient illustrer l'importance des activités non agricoles dans cette zone agro écologique.

\section{Méthodologie}

Les données utilisées proviennent de l'Enquête de Suivi de la Pauvreté au Sénégal (ESPS), effectuée en 2005. Cette enquête s'articule essentiellement autour du renseignement des indicateurs de suivi des conditions de vie et la pauvreté. Elle s'est déroulée sur tout le territoire national.

En effet, cet article met en exergue un examen de la pauvreté des actifs du secteur non agricole et de ceux du secteur agricole. Ce qui laisse entrevoir la sensibilité des ménages à une insuffisance de ressources financières par rapport à l'activité qu'ils exercent. Celle-ci a pu être définie à l'aide du calcul des ratios du nombre d'actifs déclaré « pauvre » sur celui déclaré « non pauvre », relatif à chaque activité. L'intérêt du calcul des ratios est de mettre en évidence lequel des deux secteurs serait plus bénéfique aux ruraux. En d'autres termes, il s'agit de faire ressortir le secteur d'activité qui enregistre le ratio « pauvre/non pauvre » le plus faible. 
Des auteurs comme Hayami et Godo (2004) ont fait une étude comparative des revenus du travail notamment de l'agriculture et des autres secteurs (Industrie et Services). Ils ont également utilisé une méthode de calcul des ratios précisément «le ratio de revenu du travail ».

Le ratio pour un secteur ou un sous-secteur d'activité est calculé ici en faisant le rapport entre nombres d'individus déclarés pauvres et le nombre d'individus déclarés non pauvres pour ce même secteur ou sous-secteur d'activité.

La formule générale de calcul du ratio « pauvre/non pauvre » pour chacun des secteurs d'activité, est la suivante :

(1) $\mathrm{R}=\frac{N P}{N N P}$

Avec

$\mathrm{R}=$ Ratio

$\mathrm{NP}=$ Nombre d'individus déclarés pauvres

$\mathrm{NNP}=$ Nombre d'individus déclarés non pauvres

(2) $\mathrm{R}=\frac{\text { Nombre d'individus déclarés pauvres }}{\text { Nombre d'individus déclarés non pauvres }}$

Pour chacune des activités suivantes (Agriculture, Services, Industrie), une analyse de la sensibilité à la pauvreté est fournie.

\section{Résultats}

Les résultats du tableau 1 - dans sa dernière colonne - montrent les ratios « Pauvre/Non Pauvre » pour les différents secteurs d'activités à savoir l'agriculture et les activités non agricoles (prises individuellement - c'est-à-dire Services et Industrie - d'une part et globalement d'autre part).

Tableau 1 : Etat de la pauvreté des individus selon le secteur d'activité.

\begin{tabular}{|c|c|c|c|c|c|c|c|}
\hline $\begin{array}{l}\text { Secteur } \\
\text { d'activités }\end{array}$ & $\begin{array}{l}\text { Effectif des } \\
\text { Pauvres } \\
\text { dans } \\
\text { l'échantillon }\end{array}$ & \begin{tabular}{|lr} 
Part & en \\
pourcentage \\
des pauvres \\
parmi & les \\
actifs & des \\
deux & secteurs
\end{tabular} & $\begin{array}{l}\text { Part en } \\
\text { pourcentage } \\
\text { des pauvres } \\
\text { par activité }\end{array}$ & $\begin{array}{l}\text { Effectif des } \\
\text { non pauvres } \\
\text { dans } \\
\text { l'échantillon }\end{array}$ & 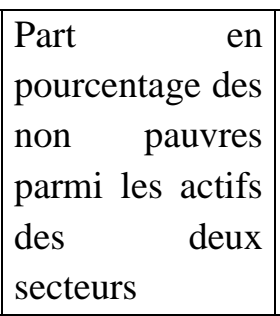 & $\begin{array}{lr}\text { Part } & \text { en } \\
\text { pourcentage } \\
\text { des } & \text { non } \\
\text { pauvres } & \text { par } \\
\text { activité } & \end{array}$ & $\begin{array}{l}\text { Ratio } \\
\text { Pauvre/Non } \\
\text { Pauvre }\end{array}$ \\
\hline Agriculture & 4655 & 50,17 & 72,45 & 1468 & 15,82 & 51,44 & 3,17 \\
\hline Services & 1428 & 15,39 & 22,22 & 1123 & 12,1 & 39,35 & 1,27 \\
\hline Industrie & 342 & 3,69 & 5,33 & 263 & 2,83 & 9,20 & 1,3 \\
\hline $\begin{array}{l}\text { Total } \\
\text { Activités } \\
\text { non } \\
\text { agricoles }\end{array}$ & 1770 & 19,08 & 27,55 & 1386 & 14,93 & 48,55 & 1,28 \\
\hline $\begin{array}{l}\text { Total } \\
\text { Général }\end{array}$ & 6425 & 69,25 & 100 & 2854 & 30,75 & 100 & \\
\hline
\end{tabular}

Sources : Base de données ESPS 
Le tableau 2 laisse entrevoir les détails du sous-secteur Industrie ainsi que le calcul du ratio «Pauvre/Non Pauvre». Les différentes activités sont composées des Bâtiments et Travaux PublicsBTP, la fabrication de produits alimentaires, les branches manufacturières, l'extraction minière et enfin les activités liées à la production et distribution de l'eau, l'électricité et le gaz. Les ratios varient de 0,83 à 1,55 .

Tableau 2 : Etat de la pauvreté du secteur Industrie.

\begin{tabular}{|l|l|l|l|l|l|}
\hline & $\begin{array}{l}\text { Effectif des } \\
\text { pauvres par sous- } \\
\text { secteur d'activité }\end{array}$ & $\begin{array}{l}\text { Part } \\
\text { pourcentage }\end{array}$ & $\begin{array}{l}\text { enfectif des non } \\
\text { pauvres par } \\
\text { sous-secteur } \\
\text { d'activité }\end{array}$ & $\begin{array}{l}\text { Ratio } \\
\text { Part en } \\
\text { pourcentage }\end{array}$ & $\begin{array}{l}\text { Pauvre/Non } \\
\text { Pauvre }\end{array}$ \\
\hline BTP & 188 & 31,07 & 121 & 20 & $\mathbf{1 , 5 5}$ \\
\hline $\begin{array}{l}\text { Fabrication de } \\
\begin{array}{l}\text { Produits } \\
\text { alimentaires }\end{array}\end{array}$ & 19 & 3,14 & 15 & 2,48 & $\mathbf{1 , 2 7}$ \\
\hline $\begin{array}{l}\text { Branches } \\
\text { manufacturières }\end{array}$ & 101 & 16,69 & 90 & 14,88 & $\mathbf{1 , 1 2}$ \\
\hline Extraction minière & 24 & 3,97 & 25 & 4,13 & $\mathbf{0 , 9 6}$ \\
\hline $\begin{array}{l}\text { Eau, électricité, } \\
\text { gaz }\end{array}$ & 10 & 1,65 & 12 & 1,98 & $\mathbf{0 , 8 3}$ \\
\hline Total & 342 & 56,53 & 263 & 43,47 & \\
\hline
\end{tabular}

Sources : Base de données ESPS

Quant au troisième et dernier tableau, il présente le sous-secteur des services composé de l'administration, du commerce, des services, du transport, de l'hôtellerie et restauration et des autres services. Les ratios « Pauvre/Non Pauvre » varient de 1,04 à 1,72.

Tableau 3 : Etat de la pauvreté du secteur des Services.

\begin{tabular}{|l|l|l|l|l|l|}
\hline Services & $\begin{array}{l}\text { Effectif des } \\
\text { pauvres par sous- } \\
\text { secteur d'activité }\end{array}$ & $\begin{array}{l}\text { Part } \\
\text { pourcentage }\end{array}$ & $\begin{array}{l}\text { Effectif des non } \\
\text { pauvres par } \\
\text { sous-secteur }\end{array}$ & $\begin{array}{l}\text { Part en } \\
\text { pourcentage }\end{array}$ & $\begin{array}{l}\text { Ratio } \\
\text { Pauvre/Non } \\
\text { Pauvre }\end{array}$ \\
\hline Administration & 74 & 2,9 & 71 & 2,78 & $\mathbf{1 , 0 4}$ \\
\hline Commerce & 843 & 33,05 & 682 & 26,73 & $\mathbf{1 , 2 4}$ \\
\hline Services & 168 & 6,59 & 118 & 4,63 & $\mathbf{1 , 4 2}$ \\
\hline Autres Services & 164 & 6,43 & 93 & 3,65 & $\mathbf{1 , 7 6}$ \\
\hline Transport & 160 & 6,27 & 148 & 5,8 & $\mathbf{1 , 0 8}$ \\
\hline $\begin{array}{l}\text { Hôtellerie } \\
\text { Restauration }\end{array}$ & 19 & 0,74 & 11 & 0,43 & $\mathbf{1 , 7 2}$ \\
\hline Total & 1428 & 55,98 & 1123 & 44,02 & \\
\hline
\end{tabular}

Sources : Base de données ESPS 


\section{Discussions}

\subsection{Agriculture}

Pour le secteur de l'agriculture, le ratio « pauvre/non pauvre » évalué ainsi à 3,17 (Tableau 1), traduit toute la difficulté de ce secteur bien qu'il continue d'employer le maximum de ménages en zone rurale. Les ménages agricoles restent trop affectés par une insuffisance de ressources financières, puisque l'activité continue de dépendre d'une pluviométrie irrégulière et imprévisible. Or, le risque de diminution des revenus des agriculteurs remet en cause selon Dupaigre (2017), l'espoir mis dans l'agriculture comme source d'emploi et facteur de croissance. D'autant plus qu'un secteur qui n'est pas capable de générer des activités rémunératrices n'attirera pas les jeunes qui arrivent en masse sur le marché du travail. Pour cet auteur, neuf (9) pays dont le Sénégal verrait les revenus de leurs agriculteurs continuer à se réduire en raison de la baisse de la productivité des terres. Par conséquent, cette baisse pourrait favoriser des conséquences néfastes, dont l'accroissement des pressions d'émigration, l'extension de la pauvreté et de la famine.

\subsection{Economie rurale non agricole}

Parmi les ruraux qui se sont déclarés pauvres, seuls 27,55\% sont occupés par l'économie rurale non agricole (Tableau 1). Les services enregistrent toutefois moins de pauvres que les métiers du secteur de l'Industrie. Seulement 5\%, soit environ $1 / 5$ des actifs non agricoles pauvres, s'exerçaient dans ce domaine. La plus faible part des pauvres est notée dans l'hôtellerie et la restauration. Cette activité enregistre au même moment le plus faible nombre de non pauvres parmi les emplois du secteur des Services, soit $0,43 \%$ (Tableau 3). Néanmoins, le ratio «pauvre/non pauvre » présente les actifs de cet emploi comme sensibles à une insuffisance de ressources financières.

Le commerce quant à lui, compte $33 \%$ de pauvres et $27 \%$ de non pauvres parmi les participants aux activités du secteur des Services. Il enregistre la part la plus importante de pauvres et de non pauvres dans tous les deux secteurs d'activités de l'économie non agricole. Son ratio de pauvreté qui s'élève à 1,24 fait de ses pratiquants, moins affectés par un manque de ressources financières contrairement à l'hôtellerie et la restauration, aux services et aux autres services. Les plus faibles ratios de pauvreté sont retenus pour l'administration et le transport. Les ratios «pauvre/non pauvre » de ces activités sont successivement de 1,04 et 1,08. Ce qui laisse entrevoir que l'exercice de ces emplois fait de leurs participants, moins affectés par une insuffisance de revenus.

Le domaine des services projette ainsi $56 \%$ de pauvres et $44 \%$ de non pauvres (Tableau 3). Le ratio de pauvreté évalué à 1,27 est inférieur à celui du secteur des Industries, qui s'élève à 1,30 et à celui de l'agriculture fixé à 3,17. Il est donc 2,5 fois inférieur à celui de l'agriculture qui demeure plus exercée par les ruraux. Par ailleurs, les études antérieures dans le domaine des services à l'image du rapport de la CNUCED (2015) ont montré que la dynamique des services a assurément contribué à la croissance qu'a connue l'Afrique au cours de la dernière décennie. Ce qui corrobore les résultats de cet article. Les services doivent en outre soutenir le processus de transformation structurelle, c'est-à-dire le passage d'activités à faible productivité à des activités à forte productivité et une diminution de la part de l'agriculture dans la production et l'emploi. Sur le plan macroéconomique également, de nombreux gouvernements africains ont surtout compris l'importance qu'avait eue l'économie des services dans la trajectoire de croissance de l'Afrique et la contribution que le secteur pouvait apporter à la création d'emplois durables et à la réduction de la pauvreté (Ibid.).

En ce qui concerne les emplois industriels, 22\% de personnes se déclarant pauvres sont occupées par des activités non agricoles (Tableau 1). D'abord, les BTP constituent l'une des principales activités hors agriculture et emploient $51 \%$ des participants à la branche Industrie, dont $31 \%$ parmi eux se sont considérés comme pauvres et $20 \%$ comme non pauvres (Tableau 2). Son ratio de pauvreté est supérieur à celui des autres activités. Autrement dit, elle est l'activité la plus susceptible de connaitre un manque 
de ressources financières. Elle est suivie par les branches manufacturières en termes d'effectif, soit $31,5 \%$ des participants dont $14,88 \%$ d'entre eux sont non pauvres. Le ratio de pauvreté estimé à 1,12 fait des exerçants de ces micros et petites entreprises moins affectés par un manque de revenus financiers par rapport à beaucoup d'autres emplois ruraux non agricoles (Tableau 2).

Deux des activités rurales non agricoles du secteur des industries en l'occurrence l'extraction minière et les activités liées à la production ou à la distribution de l'eau, de l'électricité et du gaz, laissent exceptionnellement entrevoir des ratios inférieurs à l'unité. Cela signifie que le nombre d'exerçants non pauvre de ces métiers est supérieur à ceux déclarés pauvres. Ces emplois non agricoles sont donc susceptibles à une amélioration des revenus des participants, mais également de ceux qui n'en trouvent pas mieux, ailleurs dans l'agriculture et les métiers connexes.

Le ratio de pauvreté évalué ainsi à 1,30 soit légèrement supérieur à celui des services, fixé à 1,27 fait du secteur Industrie moins sensible à une insuffisance de ressources financières par rapport à la première activité économique de la zone qu'est l'agriculture.

Globalement, les ratios plus faibles pour le secteur non agricole constituent des résultats en phase avec l'étude menée par Faye et al. (2019) sur la pauvreté et les inégalités dans le Bassin arachidier du Sénégal. Les auteurs ont montré que les revenus extra agricoles - tirés des industries et services ruraux - ainsi que les transferts creusent les inégalités et pourraient permettre une sortie massive de la pauvreté.

Cette étude montre en outre que les revenus tirés des activités extra-agricoles renforcent la capacité d'investissement et de prise de risques des ménages les plus aisés. Ces derniers peuvent se lancer dans des cultures de diversification, encore plus rentables. Ce qui signifie que les services et industries ruraux, au-delà de leur caractère de renfort des revenus du ménage, permettent une propulsion du secteur agricole en jouant le rôle de source de financement. Ces résultats sont confirmés par une recherche de la Banque mondiale réalisée en 2015. Selon cette institution, il faut augmenter les opportunités et les capacités du monde rural à bénéficier de l'économie rurale non agricole qui est essentielle pour des perspectives d'élimination de la pauvreté et de l'augmentation de la prospérité.

\section{Conclusion et recommandations}

Cet article établit par des faits stylisés un examen de la pauvreté des actifs du secteur non agricole et de ceux du secteur agricole. Il en ressort que le secteur non agricole emploie des actifs moins sensibles à la pauvreté par rapport à l'activité économique principale qu'est l'agriculture. Globalement, les ratios «pauvre/non pauvre » de 1,27 et 1,30 respectivement pour les services et industries justifient le choix des Services par les actifs non agricoles du fait qu'il présente le ratio le plus faible et donc seraient-ils moins affectés par une insuffisance de revenus. D'ailleurs, des recherches font ressortir depuis longtemps le rôle positif des activités rurales non agricoles dans la lutte contre la pauvreté. Les localités rurales, par lesquelles passent les échanges d'intrants, de biens et de services entre l'intérieur rural et les grands centres urbains, sont considérées comme les meilleurs moyens de créer des emplois non agricoles pour les pauvres.

Le secteur des Services, par exemple, serait devenu prépondérant dans certains pays africains et participerait activement à leur croissance économique. Il demeure cependant toujours informel même avec cette forte croissance qui laisse émerger des entreprises informelles.

Néanmoins, l'importance du secteur non agricole en tant que fournisseur d'emplois et de moyens de subsistance pour la main-d'œuvre en plein essor, implique leur prise en compte fondamentale dans l'élaboration de politiques publiques en faveur des zones rurales, mais aussi de lutte contre la pauvreté. Par ailleurs, les résultats susmentionnés amènent à formuler quelques recommandations pour les ménages ainsi que les politiques. Les premiers, souvent victimes d'insuffisances chroniques de revenus en raison de la seule pratique d'activités agricoles - quatre mois au plus durant l'année - doivent varier 
leurs sources de revenus en pratiquant une voire plusieurs activités non agricoles. Le choix doit en effet plus être orienté vers les services puisque présentant le ratio «pauvre/non pauvre » le plus faible. De manière spécifique, les ruraux doivent chercher à s'employer plus dans l'administration. Au-delà de cette dernière, le transport ainsi que le commerce sont les plus utiles pour ces derniers.

En outre, les politiques doivent faciliter aux individus et ménages ruraux, le démarrage de ces activités non agricoles à travers des financements et un suivi permanent des activités.

\section{BIBLIOGRAPHIE}

[1] Agence Française de Développement-Union Européenne. «Pauvreté et inégalités dans le bassin arachidier du Sénégal : implications et recommandations. DIALOGUES DE POLITIQUES PUBLIQUES. Décembre $2020-\mathrm{n}^{\circ} 16 \mid$ Facilité de recherche UE-AFD sur les inégalités ». 2020.

[2] Agence Nationale de la Statistique et de la Démographie (ANSD). Pauvreté et condition de vie des ménages. 2014.

[3] Agence Nationale de la Statistique et de la Démographie (ANSD). Recensement Général de la Population et de l'Habitat, de l'Agriculture et de l'Elevage. Rapport définitif. 2013.

[4] K. Beegle, K. and L. Christiaensen. « Accélérer la réduction de la pauvreté en Afrique. Note d'orientation de la Banque mondiale ». 2019a.

[5] K. Beegle, K. and L. Christiaensen. «Accélérer la réduction de la pauvreté en Afrique. Vue d'ensemble. Banque mondiale ». 2019b.

[6] F.J. Cabral. «Pauvreté et distribution de revenus au Sénégal : une approche par la modélisation en équilibre général calculable micro-simulé ». 2003.

[7] Conférence des Nations Unies pour le Commerce et le Développement. Rapport sur le Développement économique en Afrique : Libérer le potentiel du commerce des services en Afrique pour le commerce et le développement. 2015.

[8] B. Diagana and A. Mankor. « Agriculture durable et réduction de la pauvreté dans le Bassin arachidier du Sénégal : Résultats du Modèle Analyse Tradeoffs ». 2008.

[9] A.B. Diop. Les paysans du bassin arachidier. Conditions de vie et comportements de survie. 1992.

[10] B. Dupaigre. Revenus et Trajectoires agricoles en Afrique d'ici 2050 : Vers un trop plein d'agriculteurs ? AFD. Notes techniques n²6, Mars 2017, AFD, 2017.

[11] N.F. Faye, M. Sall, F. Affholder and F. Gérard. Inégalités de revenu en milieu rural dans le bassin arachidier du Sénégal », Papiers de Recherche AFD, n 115. 2019.

[12] Fonds International de Développement de l'Agriculture. Créer des opportunités dans l'économie rurale non agricole. Dans : Rapport sur la pauvreté rurale 2011, chapitre 6. Rome : FIDA. 2011.

[13] Y. Hayami and Y. Godo. The Three Agricultural Problems in the Disequilibrium of World Agriculture », Journal of Agriculture and Development, 3-16. 2004.

[14] Institut Sénégalais de Recherche Agricole. Caractérisation et typologie des exploitations agricoles familiales du Sénégal. Dakar, Sénégal: ISRA. 2008

[15] I. Katega. Rural Non-Farm Activities and Poverty Alleviation in Tanzania: A Case of Selected Villages in Chamwino and Bahi Districts in Dodoma Region. Presented at REPOA's 18th Annual Research Workshop held at the Kunduchi Beach Hotel, Tanzania. April 3-4. 2013.

[16] J.U. Madaki and J.O. Adefila. Contributions of Rural non-farm Economic Activities to Household Income in Lere area, Kaduna State of Nigeria. International Journal of Asian Social Science, 2014, 4(5), 654-663. 2014.

[17] Nations Unies. Evolution récente de la situation sociale en Afrique. Dans « Rapport Economique sur l'Afrique ». 2017.

[18] World Bank. Growing the Rural Non-Farm Economy to Alleviate Poverty: An IEG Evaluation of World Bank Group Support 2004-2014", Approach Paper. December 8, 2015.

[19] NDAO, A. Être mobile, seule condition pour rester au village ? Exemples dans le bassin arachidier sénégalais. Géotransports. 2017. 